\title{
An Updated Guide to Medical Assistive Devices
}

\author{
Samantha C. Wu \\ The Lawrenceville School, Lawrenceville, NJ, USA
}

\begin{abstract}
Recent technological advancements have resulted in major advances in the development of robotics and other assistive devices to improve patients' function and quality of life. The objective of this study is to provide an overview of cutting-edge assistive devices that are or will soon be available to patients with physical disabilities. For individuals with stroke or spinal cord injury, wearable exoskeletons and robotic limbs may offer hope of restoring mobility. Many of these mobility devices use miniature motors and hydraulics controlled by microprocessors to amplify the wearer' muscle strength. Features of some of the current and pipeline devices are described. Practical challenges of the exoskeletons for everyday use include the weight of the device, cost, and social acceptance. With over 300 million individuals with visual impairment worldwide, there is a large unmet need for visual neuroprosthetic devices. The first bionic eye was approved by the U.S. FDA in early 2013 for individuals with retinitis pigmentosa. An emerging neuroprosthetic technology bypasses the optic nerve and delivers electrical signals directly to the brain's visual cortex. Some of the biggest challenges of this neurovisual device include the resolution of the images as well as potential surgery-related issues. In conclusion, tremendous strides in technology have been made in the field of biomedical assistive devices. Successes and challenges of some of the latest and emerging assistive devices are discussed.
\end{abstract}

\section{Introduction}

In the past 50 years, advancements in electronics have led to a renaissance in the technological progress of assistive devices in healthcare. The innovations that compressed room-sized computers into hand-held cell phones have revolutionized the development of bioprostheses, turning science fiction into reality for individuals with physical disabilities. Some of the modern technologies with the greatest promise include robotic exoskeletons, augmentative and alternative communication devices, cochlear implants, bionic eyes, and lower extremity functional electrical stimulation cycling. The successes and challenges of these assistive devices will be discussed.

\section{Materials and Methods}

Online databases, such as Pubmed, Google Scholar and EBSCOHost, were used to perform a review of literature published through October 2014. The search was conducted in English. The search words utilized included "assistive technology", "exoskeleton", "bionic eye" and "rehabilitation".

\section{Results}

\subsection{Exoskeletons}

The first exoskeletons or wearable robots were used by soldiers at war for carrying heavy weapons and armor [1], [2]. Exoskeletons have since been applied to the rehabilitation of individuals with impaired muscle control due to injuries to the central nervous system. In particular, exoskeletons have found use in gait therapy and treadmill training. Miniature motors and/or hydraulics in the exoskeleton mimic joint movement in the human body. The exoskeleton responds to the wearer's control signals while providing stiff support and augmenting the wearer's strength. ${ }^{3}$ However, potential barriers that can limit the widespread use of exoskeletons in daily living include the heavy weight, bulky size, short battery life, and high cost.

Robotic limbs have been designed to enhance the functions of both the upper and lower limbs. These robotic limbs use external power to control the prosthetic joints via microprocessors. For example, the Boston Digital Arm System, developed by Liberating Technologies, allows the flexibility of moving along five axes and allows the arm to be customized through computer software programming [4]. The i-Limb hand (see Figure 1), developed by Touch Bionics, is the first commercially available hand prosthesis with five individually powered digits that include a manually rotatable thumb [4]. The hand offers precision, power, and key grip modes to accommodate the needs of the wearer. 


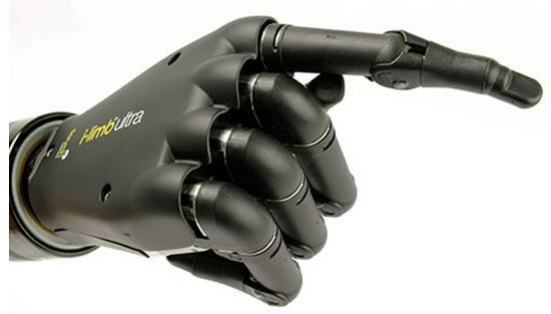

Figure 1. i-Limb Ultra

http://www.touchbionics.com/products/activ e-prostheses/i-limb-ultra

The C-Leg (see Figure 2), developed by Otto Bock Orthopedic Industry in Canada, uses hydraulic cylinders to control the joints in the prosthetic knee. The microprocessor analyzes signals transmitted by sensors in the knee and communicates information to the hydraulic cylinders to supply the necessary resistance and to position the proper angle for mobility. The C-Leg allows the amputee to walk up to 3 miles per hour, and to climb stairs [4].

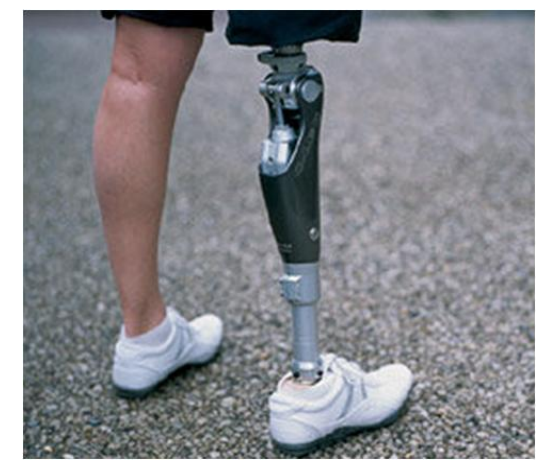

Figure 2. C-Leg

http://www.ottobock.com/cps/rde/xchg/ob_c om_en/hs.xsl/7074.html

The ReWalk (see Figure3), developed by Argo Medical Technologies, is a robotic legs system which consists of a wearable bionic suit linked to independently controlled hip and knee joint motors. ${ }^{5}$ This system is controlled by an attached computer powered by rechargeable batteries [1]. The ReWalk is controlled through subtle trunk motion and changes in center of gravity. A tilt sensor, which detects the angle of the torso, sends signals to the hip and knee joint motors to generate the appropriate stepping motion [5]. The user can also control the system remotely through a wrist pad controller [5]. The ReWalk was approved by the FDA in 2010 for use for individuals with spinal cord injuries [1], [3].

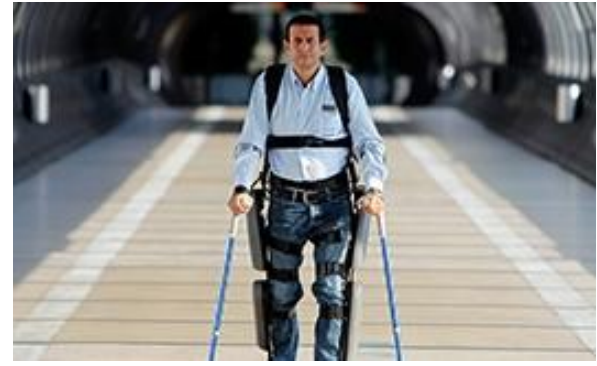

\section{Figure 3. Rewalk legs system http://rewalk.com/gallery/}

Another example of a robotic exoskeleton legs is the Rex Bionics' Rex which can enable wheelchair users to stand, walk, move sideways, turn, go up and down steps, and traverse ramps and slopes [1].

One of the exoskeleton systems that appears to hold promise is the hybrid assistive limb (HAL) system that is currently under development by companies such as Japan's Tsukuba University and the robotics company CYBERDYNE. The HAL system utilizes both an exoskeleton suit and functional electrical stimulation of the patient's muscles to take advantage of the patient's own muscle strength to reduce the energy consumption of the exoskeleton [3], [6]. Functional electrical stimulation is designed for spinal cord injury patients with weak or paralyzed muscles [6]. Further research is necessary to manage potential muscle fatigue before it can be adopted for patient use.

\subsection{Augmentative and alternative communication devices}

Augmentative and alternative communication (AAC) devices can enhance or restore the speech and writing functions of individuals with communication disorders [7]. These devices vary from a simple communication board to complex computer generated speech devices, and can be coupled with apps on smart phones, tablet computers, and other mobile devices to support their communication functions [7], [8]. Brain-computer interface (BCI) technology is under development for use as an AAC to aid those who may not have adequate voluntary muscle control to physically access these devices [8]. However, many practical issues must be overcome before BCI can become widely adopted in clinical care [9].

\subsection{Cochlear Implants}

Cochlear implants are comprised of an external component and an internal component. The external component consists of a microphone, speech processor, and transmitter [10]. The microphone detects sounds using a similar mechanism to that of a 
hearing aid. The sounds are received by the speech processor, converted to electrical impulses, and relayed to the transmitter. The transmitter sends the signals to a receiver in the internal implant, which in turn relays these signals to an electrode array embedded in the cochlea, thus artificially stimulating the auditory nerve. Because cochlear implants directly stimulate the auditory nerve, they bypass faulty structures in the ear altogether [10]. This quality serves as an advantage over the traditional hearing aid, which amplifies environmental sounds to accommodate for desensitized hearing [11]. The cochlear implant thus may serve more helpful for patients with sensorineural damage. However, hearing with the aid of a cochlear implant requires time and practice for patients to master, as hearing with a cochlear implant differs from hearing without one.

\subsection{Bionic Eyes}

The development of visual neuroprostheses has rapidly progressed in response the large unmet need for visual neuroprosthetic devices, as there are approximately 314 million visually impaired individuals worldwide [12], [13]. Visual neuroprostheses have been inspired in part by the success of cochlear implants [14].

One technology that has received much attention is a retinal-based prosthesis that employs an implanted electrode array to induce artificial vision By stimulating the surviving retinal neuronal cells, it replaces the function of the degenerated photoreceptor cells. This retinal approach may be suitable for patients with retinitis pigmentosa and age-related macular degeneration [13]. The Alpha IMS subretinal implant developed by Retina Implant AG in Reutlingen, Germany, is an example of subretinal prostheses. The implant uses a subretinal camera that that converts light in the pixels of captured images into electrical currents [15]. However, the prostheses can only restore limited vision, as all but one subject in a study reached a maximum of $20 / 1000$ vision with the aid of the Retinal Implant AG [15]. Because the power supply involves cables that must be implanted into the sclera, installation of the prostheses involves high risk associated with invasive surgery.

The bionic eye is another promising assistive device. In early 2013, the bionic eye first won FDA approval in the United States for the treatment of severe retinitis pigmentosa in patients at least 25 years of age [16], [17]. The Argus II bionic eye developed by Second Sight Medical Products (see Figure 4) has allowed individuals to recognize large letters and locate positions of household objects [13]. Future research will aim to improve the resolution of the images and decrease the size of the device.

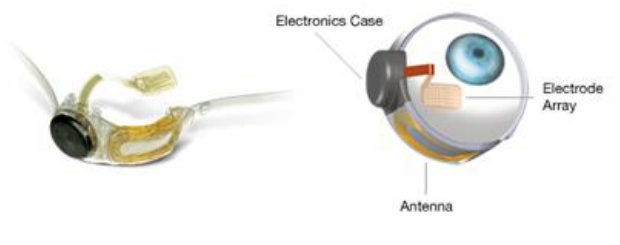

\section{Figure 4. Argus II Retinal Prosthesis System http://www.2-sight.eu/ee/system- overview}

Another approach is to bypass the optic nerve and directly stimulate the visual cortex of the brain [17]. This approach might benefit individuals who have lost their eyes or sustained trauma to their optic nerves [18].

\subsection{Lower Extremity Functional Electrical Stimulation Cycling}

Lower extremity functional electrical stimulation (FES) cycling uses electrical currents to induce muscle contractions to create movement [19]. Lower extremity FES cycling has been shown to benefit functional recovery in patients with spinal cord injury [20].

An example of lower extremity FES is the RT300 FES-LEC developed by Restorative Therapies, Inc, in Baltimore, Maryland. The RT300 FES-LEC stimulates the quadriceps, hamstrings, and gluteal muscles through surface electrodes [21]. The system's Internet connectivity feature enables easier results tracking and modification of electrical stimulation parameters. The system collects performance data, such as time, distance, power, and energy expenditure. In addition, the system allows the user to cycle from his/her wheelchair without transferring onto a separate machine. Challenges associated with the RT300 FES-LE cycle are cost and potential pain, which can act as a deterrent to cycling.

\section{Discussion}

Assistive devices, such as exoskeletons, robotic limbs and sensory aids, can dramatically improve the function and quality of life of individuals with physical disabilities. Nanotechnology and biomedical engineering are rapidly growing fields with application in assistive devices. Some of the technologic advancements in the horizon hold promise in overcoming certain practical concerns and barriers in clinical application. Aside from the obvious bioengineering challenges, such as durability and hydraulic power, future research should address potential social barriers for 
acceptance of these devices. For example, a cosmetically appealing prosthetic is likely to be more attractive and socially acceptable than a prosthetic with a cold clinical appearance that draws negative attention an individual's disability [15].

\section{Conclusion}

Technological development of assistive devices has matured rapidly in the last 10 years and will likely continue to evolve in the coming years. As research progresses, this field will probably experience a greater variety and improved costeffectiveness of devices. The resulting increase in availability of assistive devices will improve the lives of individuals with physical disability.

\section{References}

[1] "Powered exoskeleton", http://en.wikipedia.org/ wiki/Powered_exoskeleton, cited 2014 May 26.

[2] R.E Cowan, B.J. Fregly, M.L.Boninger, L.Chan , M. M. Rodgers, and D.J. Reinkensmeyer, "Recent trends in assistive technology for mobility", NeuroEng and Rehabil, 2012 Sept 20.

[3] P. Sale, M. Franceschini, A. Waldner, S. Hesse, "Use of the robot assisted gait therapy in rehabilitation of patients with stroke and spinal cord injury," Eur J Phys Rehabil Med, 2012, pp. 111-21.

[4] "Prosthesis", http://en.wikipedia.org/wiki/ Prosthesis, cited 2014 May 26.

[5] A Esquenazi, M. Talaty, A. Packel, and M. Saulino, "The ReWalk Powered Exoskeleton to Restore Ambulatory Function to Individuals with Thoracic-Level Motor-Complete Spinal Cord Injury", Am J Phys Med Rehabil, 2012 Nov, pp. 911921

[6] A.J. del-Ama, A.D. Koutsou, J.C. Moreno, A. delos-Reyes, A. Gil-Agudo, and J.L.Pons, "Review of hybrid exoskeletons to restore gait following spinal cord injury." J Rehabil Res Dev, 2012, pp. 497-514.

[7] D.E. Thompson, S. Blain-Moraes, and J.E. Huggins, "Performance assessment in braincomputer interface-based augmentative and alternative communication", Biomed Eng Online, 2014, pp.43.

[8] S. Fager, L. Bardach, S.Russell, and J. Higginbotham, "Access to augmentative and alternative communication: new technologies and clinical decision-making", J Pediatr Rehabil Med, 2012; pp. 53-61.
[9] I. Daly, M. Billinger, J. Laparra-Hernández, F. Alois, M.L. García, J. Faller, R. Scherer, G. Müllerand Putz, "On the control of brain-computer interfaces by users with cerebral palsy", Clin Neurophysiol, 2013.

[10] "Cochlear Implants", http://www.asha.org/ public/hearing/Cochlear-Implant, cited 2014 May 26.

[11] "Cochlear Implants", http://www.nidcd.nih.gov/ health/hearing/pages/coch.aspx, cited 2014 May 26.

[12] "Building the Bionic Eye: An Emerging Reality and Opportunity", http://www.ncbi.nlm.nih.gov/pmc/articles/PMC3326 660/, cited 2014 May 26.

[13] "Visual impairment and blindness", http://www.who.int/mediacentre/factsheets/fs282/ en/cited 2014 May 26.

[14] J.K. Niparko and R. Blankenhorn, "Cochlear implants in young children", Ment Retard Dev Disabil Res Rev, 2003, pp. 267-75.

[15] D. Palanker, "Substitutes for Lost Photoreceptors", The Scientist, 2014 Oct, pp 36-37

[16] "FDA Approves Argus II Bionic Eye from Second Sight", http://www.medgadget.com/2013/02/ new-post.html, cited 2014 May 26.

[17] "FDA Approves 'Bionic Eye' to Help Against Rare Vision Disorder", http://health.usnews.com/ health-news/news/articles/2013/02/14/fda-approvesbionic-eye-to-help-against-rare-vision-disorder, cited 2014 May 26.

[18] "The Bionic Eye", http://www.youthcentral. vic.gov.au/ViewPage. action?siteNodeId=1993 \&repositoryName $=$ www.youthcentral $\&$ CurrentFolde rID=1966\&ItemID=15746\#.UaqFo8zD-cw, cited 2014 May 26.

[19] J. Unterreiner, "The Effect of the FES Bike on Muscle Function and Histology in Patients Post-SCI: An Evidence Based Review", May 5.

[20] C. L. Sadowsky, E. R. Hammond, A. B. Strohl, P. K. Commean, S. A. Eby, D. L. Damiano, J. R. Wingert, K.T. Bae, and J. W. McDonald J.W. "Lower Extremity Functional Electrical Stimulation Cycling Promotes Physical and Functional Recovery in Chronic Spinal Cord Injury." J Spinal Cord Med, 2014, pp.623-631.

[21] D.R. Dolbow, A.S. Gorgey, J.M. Ketchum, J.R. Moore, L.A. Hackett, and D.R. Gater, "Exercise Adherence During Home-Based Functional lectrical 
International Journal of Technology and Inclusive Education (IJTIE), Special Issue Volume 1, Issue 2, 2014

Stimulation Cycling by Individuals with Spinal Cord Injury", Am J Phys Med Rehabil, 2012 Nov, pp 922930

[22] "Snake arms and crystal legs: Artificial limbs push boundaries of art", http://www.cnn.com/ 2013/04/24/world/europe/alternative-limb-project. cited 2014 May 26. 